\title{
Recipes for ULX formation: necessary ingredients and garnishments
}

\author{
Roberto Soria ${ }^{1,2}$ \\ ${ }^{1}$ Harvard-Smithsonian CfA, 60 Garden st, Cambridge, MA 02138, USA \\ email: rsoria@cfa.harvard.edu \\ ${ }^{2}$ MSSL, University College London, Holmbury St Mary, Dorking, RH5 6NT, UK
}

\begin{abstract}
I summarize the main observational features that seem to recur more frequently in the ULX population. I speculate that two of the most important physical requirements for ULX formation are low metal abundance, and clustered star formation triggered by external processes such as molecular cloud collisions. In this scenario, most ULX are formed from recent stellar processes, have $\mathrm{BH}$ masses $<100 M_{\odot}$, and do not require merger processes in super star clusters.
\end{abstract}

Keywords. black hole physics - X-ray: binaries — galaxies: star clusters

\section{Common features in the ULX population}

The nature and formation mechanisms of ULXs remain unclear. Much of the uncertainty is due to the lack of direct mass estimates of the compact objects that power ULXs. It is difficult to unequivocally identify or take spectra (let alone phase-resolved spectra) of their optical counterparts, at distances $\gtrsim$ a few Mpc. This prevents the determination of ULX mass functions. Moreover, ULXs are an ill-defined class of systems, based simply on their apparent luminosity: they may include diverse physical objects. Nonetheless, it is possible and useful to summarize common features that appear associated with a majority of ULXs, to find out which of those phenomena are a clue to their physical nature. Here, we discuss some of them, with particular attention to the brightest ULXs (i.e., those with X-ray luminosities $\gtrsim 10^{40} \mathrm{erg} \mathrm{s}^{-1}$, of which only $\sim 20$ are known).

- Do ULXs require an accreting black hole (BH)? In most cases, YES. Some young supernova remnants (SNRs) can be misidentified as ULXs (e.g., Circinus Galaxy $\mathrm{X}-2$ ). So can young high-energy pulsars, with magnetic fields $\sim 10^{14}$ Gauss and rotational periods $\sim 10 \mathrm{~ms}$ (Stella \& Perna 2004); they are also likely to be associated with young SNRs. But most ULXs show long-term fluctuations and flux variability inconsistent with the SNR or pulsar model, and lack X-ray emission lines, unlike typical SNR spectra.

- Do they require BHs more massive than typical Galactic BHs? That is, with masses $>20 M_{\odot}$ ? Probably $Y E S$. Their apparent luminosity is up to 50 times higher, and at least a few of them are inconsistent with strong beaming. Models explaining such a high enhancement entirely with super-Eddington emission or beaming are not ruled out yet, but the simplest scenario consistent with the observations is to allow for higher $\mathrm{BH}$ masses. If so, it means that the main difference between ULXs and Galactic BHs is due to the compact object, rather than the companion star or the gas flow.

- Do they require intermediate-mass BHs? That is, with masses $\gtrsim 200 M_{\odot}$ ? This is a controversial issue. In the absence of kinematic masses, various indirect methods have been suggested: X-ray spectral modelling, timing analysis, breaks in the luminosity function, patterns of state transitions, X-ray-radio correlations. Timing features such as low-frequency QPOs and breaks in the power-density spectrum suggest masses either one or two orders of magnitude higher than stellar-mass BHs, depending on the assumed 
model. The break or cutoff in the ULX luminosity function at $\approx 3 \times 10^{40} \mathrm{erg} \mathrm{s}^{-1}$ (Swartz et al. 2004; Gilfanov et al. 2004) suggests an upper mass limit $\approx 200 M_{\odot}$ if the Eddington limit is adhered to, or less if super-Eddinton emission is allowed.

The presence of a soft-excess in the X-ray spectra of the brightest ULXs, with a characteristic temperature $\approx 0.15 \mathrm{keV}$, was interpreted as evidence in favor of $\mathrm{BH}$ masses $\sim 1000 M_{\odot}$, if the emission comes from a standard accretion disk (Miller et al. 2004). However, we argued (Soria et al. 2006) that that argument is incorrect: when the dominant power-law component is also taken into account, the luminosity and temperature are consistent with masses $\sim 50 M_{\odot}$. It is also possible that the soft excess does not come from a disk, but from reprocessing in an ionized outflow (Gonçalves \& Soria 2006).

In conclusion, the available observational evidence does NOT require intermediate-mass BHs with masses $\sim 1000 M_{\odot}$ (although they are not ruled out, either), and is still consistent with masses $\lesssim 100 M_{\odot}$. Stellar-evolution models predict that He cores with masses $64 M_{\odot} \lesssim M \lesssim 133 M_{\odot}$ are disrupted by the pair instability and do not collapse into a $\mathrm{BH}$ (Heger \& Woosley 2002; Yungelson 2006). Therefore, there might be two subclasses of ULXs: one with BH masses $\lesssim 70 M_{\odot}$ (accounting for some mass increase due to accretion) and one with masses $\gtrsim 130 M_{\odot}$. There is no observational evidence of such dichotomy, and it is likely that most ULXs belong to the lower-mass group. However, a few (4 or 5) ULXs have been observed at least once with apparent luminosities $\sim 5-12 \times 10^{40} \mathrm{erg}$ $\mathrm{s}^{-1}$ and have been labelled "hyperluminous X-ray sources" by some authors. One may speculate that they belong to the higher-mass group; alternatively, some of them could be nuclear BHs of disrupted satellite galaxies (King \& Dehnen 2005).

- Do they require young stellar environments? YES. Most ULXs brighter than a few $10^{39} \mathrm{erg} \mathrm{s}^{-1}$ are located in star-forming environments, rather than spiral bulges, halos and elliptical galaxies. This suggests that ULXs are scaled-up versions of high-mass X-ray binaries, with an OB donor star overflowing its Roche lobe. This enables a mass transfer rate $\gtrsim 10^{-6} M_{\odot} \mathrm{yr}^{-1}$ for a few Myr (nuclear timescale), and suggests characteristic ULX ages $\sim 10^{7}$ yr. Low-mass donors could reach this level of mass transfer only during short-lived (thermal timescale) evolutionary phases, at a much later age ( $>1 \mathrm{Gyr}$ ).

- Do they require a donor star in a binary system? Probably YES. Models based on Bondi accretion from molecular clouds (Krolik 2005) cannot be ruled out in some cases, but are generally disfavoured by the low absorption seen in almost all ULX spectra (typically $<10^{21} \mathrm{~cm}^{-2}$ ) and low extinction in the surrounding stellar population. It is unlikely that all ULXs accreting from molecular clouds are at the very edge of them.

- Do they require starburst environments? NO. Although starburst conditions are positively correlated with ULX formation, they do not seem to be a necessary condition. Some of the brightest ULXs are located in dwarf irregular galaxies with only localized star formation (such as those in Ho II and Ho IX, in the M 81 group). Others are located in normal star-forming (not starburst) galaxies, such as NGC 1313 and NGC 1365. A few are in relatively quiescent environments of starburst galaxies (for example, the two brightest ULXs in NGC 7714), many kpc away from the starburst region.

- Does ULX formation require super-star-clusters (SSCs)? NO. Very few ULXs are found in SSCs, defined as young, compact clusters with stellar masses $\gtrsim$ a few $10^{5} M_{\odot}$ and sizes $\lesssim$ a few pc. Among ULXs with $L_{\mathrm{X}}>10^{40} \mathrm{erg} \mathrm{s}^{-1}$, the only examples are one in M 82 and one in NGC 7714; none are found in the Antennae; there may be some in the Cartwheel but it is too far for unequivocal identifications. In most cases, ULXs are near or inside OB associations or small open clusters, with no SSCs nearby. Characteristic stellar ages $\left(\sim 10^{7} \mathrm{yr}\right)$ are too young to be consistent with the evaporation of a hypothetical parent SSC. Even if we assume that a parent cluster had 
time to disperse, the integrated mass of all the stars seen today within $\sim 100$ pc of a ULX does not generally add up to $10^{5} M_{\odot} ;$ more typical values are $\lesssim 10^{4} M_{\odot}$. Finally, when SSCs and ULXs are present in the same region, typical displacements are too large (a few hundred pc) to be consistent with cluster ejection.

SSCs were modelled as an ideal environment to form BHs as massive as $\sim 500-1000 M_{\odot}$ in the local universe, via runaway core-collapse and merger of O stars over a timescale $\lesssim 3$ Myr (Portegies Zwart \& McMillan 2002). However, we have argued that there is no longer a compelling need to invoke intermediate-mass BHs in ULXs, and that the upper mass limit is likely to be somewhere between 50 and $200 M_{\odot}$. Correspondingly, if dynamical collapse and merger processes are still needed to form a very massive stellar progenitor $\left(>100 M_{\odot}\right)$, clusters as small as $\sim 10^{4} M_{\odot}$ may do the job. We have also argued (Soria 2006) that collapse and merger processes can be more efficient at an earlier stage of cluster evolution, when its protostars are still surrounded by large, optically-thick envelopes, and are still accreting from neutral intracluster gas. Collapsing molecular clumps with masses $\sim 10^{4} M_{\odot}$ are large enough to enable the formation of stars with masses $>100 M_{\odot}$ via accretion and coalescence, and at the same time are small enough to disperse quickly after the most massive stars reach the main sequence (Kroupa \& Boily 2003), leaving behind an open cluster or OB association, in agreement with the observations.

- Does ULX formation require low metal abundance? Almost certainly YES. This is supported both by (still sketchy) empirical evidence and theoretical arguments. We leave a detailed discussion of the available metallicity data for ULX host galaxies to further work. It is of course more difficult to produce BHs at higher metallicities, because more mass is lost from the progenitor star via stellar winds. At solar-metallicity, all $\mathrm{O}$ stars - including the Pistol star and $\eta$ Carinae, despite their initial masses $\approx 150-200 M_{\odot}$ - are predicted to leave behind only a neutron star. At $Z \sim 0.1$, they may produce BHs with masses $\sim 50 M_{\odot}$.

- Does ULX formation require primordial abundances? Probably NOT. Massive Pop-III stars were suggested as an alternative to local SSC scenarios for IMBH production. There may well be Pop-III BH remnants with masses up to $\sim 1000 M_{\odot}$ floating around in galactic halos, or slowly sinking towards the centres, but this does not explain the observed ULX correlation with young, star-forming environments. The PopIII scenario requires that floating $\mathrm{BH}$ remnants capture an $\mathrm{OB}$ star while they cross a star-forming environment, perhaps after being thrown out of their halo orbits during tidal interactions and collisions. In the absence of independent evidence for the very existence of Pop-III remnants, it remains an unlikely (though interesting) conjecture, especially if IMBHs are not needed after all to explain the ULX luminosity.

- Is ULX formation directly favoured by tidal interactions and collisions? Apparently YES. Many ULXs are found in tidal dwarfs, or colliding galaxies, or dwarf irregular galaxies located in tidally interacting groups. Spectacular examples include galaxies such as the Antennae, the Mice, the Cartwheel, NGC 7714/15, NGC 4485/90, and the M 81/M 82 group. Other bright ULXs are associated with local collisional events: NGC $4559 \mathrm{X}-1$ is in a ring of star formation (age $\sim 20 \mathrm{Myr}$ ) probably caused by a small satellite galaxy splashing through the gas-rich disk; M 99 X-1 is apparently located where a large, fast HI cloud is impacting the outer disk; NGC 1313 may have undergone a recent collision with a satellite, near its ULX X-2.

Are these chance associations? One simple explanation could be that collisions enhance star formation, and a high star-formation rate (SFR) leads to more X-ray binaries and a larger probability to form ULXs - the normalization of the high-mass X-ray binary luminosity function being proportional to the SFR (Gilfanov et al. 2004). While this is probably part of the explanation, it cannot the whole story. In various cases, the local 
SFR in a collisional or tidal feature is small, compared with the SFR in the rest of the galaxy or group; and yet ULXs seem to be directly associated to those environments (NGC 7714, M 99 and NGC 4559 are striking examples). I suggest that there can be a direct physical association between collisions and ULX formation, if collisions tend to produce a qualitatively different kind of star formation, that is more likely to lead to the formation of relatively massive BH remnants, and hence to some ULXs.

\section{Outlining a plausible ULX scenario}

Taking into account the previous arguments, I speculate that the following line of investigation appears the most promising. Most ULXs contain BHs with masses $\sim 50 M_{\odot}$ and in any case $\lesssim 100 M_{\odot}$, formed via direct core collapse from very massive stellar progenitors, and accrete from an $\mathrm{OB}$ star coeval with the BH progenitor. The luminosity enhancement with respect to Galactic BHs can be explained with a factor of $\approx 5-10$ in mass, and $\approx 5$ in super-Eddington emission, particularly outside the disk plane.

Progenitor stars with initial masses $\sim 150-200 M_{\odot}$ do exist (although they are very rare), and can be formed in clustered environments, via fast gas accretion and mergers of smaller protostars - this is also the way ordinary $\mathrm{O}$ stars are thought to form. The protocluster NGC $2264 \mathrm{C}$ in the Cone nebula is a textbook example of a molecular clump, with a gas mass $\approx 1700 M_{\odot}$ that is undergoing dynamical collapse (infall of $\gtrsim 10^{-3} M_{\odot}$ $\mathrm{yr}^{-1}$ over a free-fall timescale of $\sim 10^{5} \mathrm{yr}$ ) rather than turbulent fragmentation (Peretto et al. 2006). There is no need for such clusters to be more massive than $\sim 10^{4} M_{\odot}$.

Such global collapses occur when star formation is triggered by an external pressure wave. Cloud-galaxy or galaxy-galaxy collisions provide ideal environment for triggered star formation and therefore also for massive stellar progenitors. Low metal abundance provides the second ingredient, ensuring that a massive $\mathrm{BH}$ remnant is formed.

The normalization of the high-mass X-ray binary luminosity function, and probably also the number of fainter ULXs with luminosities $\sim$ a few $10^{39} \mathrm{erg} \mathrm{s}^{-1}$, is directly proportional to the SFR. However, the location of the upper-luminosity break, and hence the probability of forming ULXs with luminosities $>10^{40} \mathrm{erg} \mathrm{s}^{-1}$, depends more strongly on the two factors mentioned above: external triggers and low metal abundance. A key observational test would be to map the presence of very massive stars in nearby galaxies, although it may be difficult to distinguish them from unresolved stellar groups.

\section{References}

Gilfanov, M., Grimm, H.-J. \& Sunyaev, R. 2004, Nucl. Phys. Suppl. 132, 369

Gonçalves, A.C. \& Soria, R. 2006, Mon. Not. Royal Astron. Soc. 371, 673

Heger, A. \& Woosley, S.E. 2002, Astroph. J. 567, 532

King, A.R. \& Dehnen, W. 2005, Mon. Not. Royal Astron. Soc. 357, 275

Krolik, J.H. 2004, Astroph. J. 615, 383

Kroupa, P. \& Boily, C.M. 2002, Mon. Not. Royal Astron. Soc. 336, 1188

Miller, J.M., Fabian, A.C. \& Miller, M.C. 2004, Astroph. J. Lett 614, 117

Peretto, N., André, P. \& Belloche, A. 2006, Astron. \& Astroph. 445, 979

Perna, L. \& Stella, R. 2004, Astroph. J. 615, 222

Portegies Zwart, S.F. \& McMillan, S.L.W. 2002, Astroph. J. 576, 899

Soria, R. 2006, in: E.J.A. Meurs \& G. Fabbiano (eds.), Populations of High Energy Sources in Galaxies, IAU Symp. 230 (Cambridge: Univ. Press), p. 473 (astro-ph/0509573)

Soria, R., Gonçalves, A.C. \& Kuncic, Z. 2006, in: The multicoloured landscape of compact objects and their explosive progenitors, Cefalú, Sicily (June 2006), AIP, in press

Swartz, D.A., Ghosh, K.K., Tennant, A.F. \& Wu, K. 2004, Astroph. J. Suppl. 154, 519

Yungelson, L. 2006, these Proceedings (astro-ph/0610021) 